\title{
Personalized support for chronic conditions
}

\section{A novel approach for enhancing self-management and improving lifestyle}

Irene Lasorsa'; Pierluigi D’Antrassi'; Miloš Ajčević'; Kira Stellato2,3; Andrea Di Lenarda2,3; Sara Marceglia'; Agostino Accardo'

${ }^{1}$ Department of Engineering and Architecture, University of Trieste, Via Valerio 10, Trieste, Italy;

${ }^{2}$ Cardiovascular Center, Health Authority $n^{\circ} 1$, Via Slataper 9, Trieste, Italy;

${ }^{3}$ Department of Medicine, Surgery and Health Sciences, University of Trieste, Strada di Fiume 447, Trieste

\section{Keywords}

Patients with chronic illness or special needs, mHealth, mindfulness, decision support system, patient self-care, home care and e-health

\section{Summary}

Objective: Solutions for improving management of chronic conditions are under the attention of healthcare systems, due to the increasing prevalence caused by demographic change and better survival, and the relevant impact on healthcare expenditures. The objective of this study was to propose a comprehensive architecture of a mHealth system aimed at boosting the active and informed participation of patients in their care process, while at the same time overcoming the current technical and psychological/clinical issues highlighted by the existing literature.

Methods: After having studied the current challenges outlined in the literature, both in terms of technological and human requirements, we focused our attention on some specific psychological aspects with a view to providing patients with a comprehensive and personalized solution. Our approach has been reinforced through the results of a preliminary assessment we conducted on 22 patients with chronic conditions. The main goal of such an assessment was to provide a preliminary understanding of their needs in a real context, both in terms of self-awareness and of their predisposition toward the use of IT solutions.

Results: According to the specific needs and features, such as mindfulness and gamification, which were identified through the literature and the preliminary assessment, we designed a comprehensive open architecture able to provide a tailor-made solution linked to specific individuals' needs. Conclusion: The present study represents the preliminary step towards the development of a solution aimed at enhancing patients' actual perception and encouraging self-management and selfawareness for a better lifestyle. Future work regards further identification of pathology-related needs and requirements through focus groups including all stakeholders in order to describe the architecture and functionality in greater detail.

Correspondence to:

Irene Lasorsa

Department of Engineering and Architecture, University of Trieste,

Via Valerio 10,

Trieste, Italy

Email: irene.lasorsa@phd.units.it
Appl Clin Inform 2016; 7: 633-645

http://dx.doi.org/10.4338/ACl-2016-01-RA-0011

received: January 18, 2016

accepted: May 2, 2016

published: July 6, 2016

Citation: Lasorsa I, D'Antrassi P, Ajčević M, Stellato K, Di Lenarda A, Marceglia S, Accardo A. Personalized support for chronic conditions: a novel approach for enhancing self-management and improving lifestyle. Appl Clin Inform 2016; 7: 633-645

http://dx.doi.org/10.4338/ACl-2016-01-RA-0011 


\section{Introduction}

In their everyday lives, patients with chronic diseases deal with a range of conditions that require self-management and relevant decision-making. The attention to chronicity is acquiring worldwide relevance also because of the steadily increase in associated costs due to demographic changes and better survival rates of patients with chronic conditions [1]. The reduction of costs associated to the management of chronicity is one of the main challenges healthcare systems have to face.

Upon hospital discharge, the multidimensional aspects of patient's care converge into a combination of clinical conditions (co-morbidities, reduced functional capacity and self-sufficiency, depressive and anxiety symptoms), psychological needs (awareness, acceptance, redefinition of self, self-efficacy, empowerment), and social challenges (loneliness, family/social support) which may ultimately significantly affect patient's adherence and well-being [2].

Several approaches are currently being adopted to promote and sustain patient's adherence; among them, there is patient education (cognitive-behavioral individual and/or group interventions [3], nurse interventions, workshops, printed material, online communication); patient reminders (e-mails, tele-calling, text messages); pharmacy-based programs; collaboration with patient organizations, etc. Research has shown that the multifaceted aspects of adherence require multiple tactics to prove successful in time [4]. Between 2013-2016, Friuli Venezia Giulia Region has been leading a EU-funded, 24-region project on ICT-supported integrated care to provide domiciliary care to fragile, elderly European citizens. The experience has shared light on the needs and the maturity level of elderly citizens in the learning and use of technology and has highlighted patients' willingness to be actively engaged with their healthcare/social care teams in the management of their own health. Preliminary results show the empowering aspect of technology for senior citizens [5].

The implementation of mobile technologies may support a healthcare redesign based on disease management programs and integrated care models [6-9]. Furthermore, mHealth technologies may act as a complementary tool to provide support and motivation to regular patient's self-monitoring of health parameters (e.g., blood pressure, heart rate, blood glucose) [10]. The resulting empowerment may help patients to achieve personal health objectives, modify lifestyle patterns and/or highrisk behaviors for an optimal management of their chronic condition [11].

Currently, over 35,000 mHealth apps for different health conditions are available for iOS and Android platforms [12]. Nevertheless, many challenges are still to be faced, such as open architecture $[13,14]$, medical devices directive applicability and data protection $[15,16]$, interoperability and integration in existing healthcare models $[14,17]$. Most mHealth solutions rely on an architecture that only addresses one specific disease and are usually either all-online or all-offline [18]. Furthermore, these applications have been developed independently as stand-alone applications and are not easily integrated into existing healthcare models. Success of mHealth solutions depends also on their ability to address and meet users' needs. The diversity of needs and requirements comes from different age, sex, pathology, health status, environment and professional and social activities. The adaptability of the system in terms of customization and personalization is of paramount importance. Moreover, among others, health-related data are one of the most sensitive issues, and they are being protected by laws and regulations. However, it is well known that digital data, especially in a mobile environment, pose a huge security risk in terms of privacy violation [18]. The protection of health data is still a mHealth burning issue, which has to be handled properly. Data collected by sensors and coming from other sources do not impact only on storage and transfer capacity, but also require the ability of the system to analyze a large amount of information. Clinicians and patients are not interested in single data, rather in trend and overall scenarios. Therefore, the development of an intelligent system for data analysis and Decision Support Systems (DSSs) is essential for helping patients along their clinical pathways [19].

Many DSSs are available in current literature, but they are seldom based on validated predictive models and even though some scoring systems have been validated (e.g., Seattle Heart Failure Score [20], Archimedes [21]), predictive models are not often used in clinical practices.

Moreover, beyond the above-mentioned considerations, a crucial aspect needs to be tackled anytime an IT solution has to be developed, i.e. the psychological and social implications of the new technology from the patient's point of view. In order to answer these needs, a suitable approach must 
be found for meeting three major requirements: increasing self-management, self-awareness and social inclusion of patient/s within their communities.

Actually, self-management may reduce the number of severe events (e.g., hypoglycemia and glycemic decompensation due to hyperglycemia in case of diabetic patients), thanks to the active role played by patients in their care plan. Furthermore, educational programs concerning, for example, self-management education, acquisition of the fundamental skills to correctly interpret the symptoms and to autonomously take the relevant corrective actions are of paramount importance [22].

Secondly, self-awareness is being considered increasingly important. Self-monitoring has shown to enhance self-awareness and plays a substantial role in most behavioral programs aimed at promoting patient's adherence to therapy [23]. Research shows that people judge a risk not only by what they think about it but also by how they feel about it. If their feelings toward an activity are favorable, they tend to judge the risks as low and the benefits as high; if their feelings toward the activity are unfavorable, they tend to make the opposite judgment, i.e. high risk and low benefit [24]. Interventions aimed at improving user's risk perception need to draw their rationale on the understanding of the psychological mechanisms and relevant theories underlying human behavior and motivation, in order to bring effective and long-term outcomes.

Finally, social inclusion plays a key role in understanding, accepting and handling the complexities of change which are inherent to a life-limiting chronic illness. [25]

In this process, positive communication is essential: in fact, whilst perceived negative change may lower self-esteem and hinder self-care, positive handling of change may increase self-awareness, selfworth and boost self-management skills.

In order to overcome the limitations presented in this first section (i.e., related to open architecture, interoperability and integration, compliance with medical device directives and data protection, customization and personalization, DSSs based on validated predictive models as well as psychological and social implications), in this study we propose a comprehensive solution that integrates both technical and psychological/clinical needs through a novel approach aimed at boosting the active and informed participation of patients in their care process, allowing them to manage their own health and increase their awareness.

Namely, the present study is structured as follows. In the following section, we describe our approach focusing on clinical, psychological and social aspects to be implemented in order to overcome the related aforementioned limitations. Moreover, "Our approach" section includes a preliminary assessment which involved 22 patients attending an outpatient Chronic Care clinic. In the "Architecture" section, we present the technical requirements and architecture of our solution, focusing on the technical aspects to be considered in order to overcome the aforementioned technical limitations. In the last section, we present conclusions and future developments.

\section{Our approach}

In this section the pillars of our approach are reported, in accordance with the aforementioned clinical, psychological and social needs (e.g., patien's self-management, self-awareness, empowerment, social inclusion, positive communication). Our solution targets patients affected by the most frequent chronic conditions with no severe mental and physical disabilities.

\subsection{Mindfulness}

Our solution answers the need for increasing self-awareness through the integration of mindfulness. Mindfulness has been defined as a kind of non-judgmental, present-centered awareness in which each thought, feeling, or sensation arising within the perimeter of our attentional field is acknowledged and accepted [26]. Within this process, the person moves toward a state in which one is fully observant of internal and external stimuli in the present moment, and open to accepting rather than trying to change or judge whatever arises to their conscience [27]. Baer [28] suggests that mindfulness may promote exposure to previously avoided internal experiences, thus promoting cognitive change or a shift in attitude about one's thoughts, while at the same time increasing acceptance. According to Teasdale and colleagues [29], mindfulness practice may increase metacognitive aware- 
ness, a process through which negative thoughts and feelings are experienced as mental events rather than as the self. Moreover, a mindfulness-based approach has been incorporated within dialectical behavior therapy [30]; the combination of training and implementation of mindfulness meditation with cognitive therapy has been seen to significantly reduce relapse rates in recurrent major depression [31].

Mindfulness goes beyond the simple practice of meditation; in fact, meditation practice is simply a "scaffolding" used to develop the state, or skill of mindfulness [32]. Meditation is also often recommended as a practice that can be applied as a stress-reduction procedure to deal with a variety of health-related problems such as pain management, hypertension, and cardio-vascular diseases [33]. There is an increasing number of studies of acceptance, mindfulness, and values-based action in relation to chronic pain. Also, whilst cognitive-behavior therapy (CBT) has proved its effectiveness in treating depression and anxiety disorders [34], a 2012 Cochrane review [35] outlined that only $38-77 \%$ of patients suffering from chronic pain responded to CBT by experiencing clinically significant relief. Mindfulness-Based Cognitive Therapy (MBCT) may thus prove effective by its four-stage therapeutic approach which is designed to help people modify their thought process rather than the mere content of their thoughts [36]. Within a beehive person-centered approach [5], whereby each person is at the center of an integrated process of mutual care, mindful empowerment may thus turn out to be a pivotal issue in the development of innovative solutions for patients with chronic disease.

The proposed solution supports a mindfulness-based intervention through a tailor-made path so as to allow beginners to incorporate mindfulness practices in their everyday lives. As with any other intervention, mental health pre-screening and regular supervision need to be carried out by the clinician so as to rule out the presence of mental disorders which may put the patient at risk. The specific path (in terms of "entry level") is evaluated by administering the Five-Facet Mindfulness Questionnaire [28] to the patient, designed to assess a core characteristic of dispositional mindfulness [37].

This approach takes into consideration specific patient's needs allowing for the system personalization. The overall of the path to follow is listed below:

- Awareness of the five senses;

- Visualization exercises (e.g., bodyscan);

- Mini-guided meditation sessions;

- Daily reminders to bypass the risk of alarm fatigue so as to promote adherence;

- A motivational calendar to enhance self-care and prosocial behavior;

- The user will become part of a mindful community which reinforces each other's behaviors and enhance both individual's and social healthcare responsibility;

- Successful adherence and behavior modification will be signaled to the user through easily readable health indicators which will provide cognitive and emotional reinforcement.

\subsection{Piazza Grande}

The proposed solution enhances the importance of social inclusion of patients affected by chronic conditions in the real life through the implementation of Piazza Grande. Piazza Grande is a virtual meeting point where calendars of city health-promoting events can be shared, thus providing a means to meet in real life and share experiences (e.g., diaries and pictures) with those care recipients who are unable to join due to their serious health conditions ( Figure 1). Particularly, Piazza Grande serves as a gateway to all the different people who matter to the patient/user and also connects individuals with the same conditions. Several interactions can be made among subjects to improve engagement and reduce loneliness in some scenarios. Mindfulness may be promoted and enhanced within the virtual group of users, by sharing not only experiences, but also moments of practice and mutual support along the lines of social learning [38]. Not only patients can access the Social Network Piazza Grande, but also all the people in their "real-life network" can be part of it, such as healthcare professionals, relatives, friends, caregivers. Piazza Grande may also benefit from the support provided by the Third Sector (e.g., college students enrolled in medical and/or nursing schools) who may, in turn, learn better and more effective ways to take care of their own health and well-being. This may actually promote healthier lifestyles among a wider community of 'healthy' users. To this end, different access levels will be defined in order to guarantee the privacy of the pa- 
tient. In a person-centered solution, patients can be connected to the people who care for their health and who can help them learn self-management and accountability skills.

\subsection{Gamification}

Our approach is based on gamification to promote patients' self-management and enhance their empowerment. Indeed, it has been proved that gamification has a good impact on motivating users' behaviors; it also plays a crucial educational role, since motivation is one of the basic conditions to learn. Moreover, it has also been proven successful for empowering patients to adopt healthy lifestyle [39]. Gamification mechanics are already represented in several mHealth applications and are an encouraging implementation for incentivizing improved patient self-management [40, 41]. It has also been reported that gamification improves engagement, compliance to therapy and learning [42, 43]. Furthermore, gamification alone or with social support may increase physical activity and empowerment and decrease healthcare utilization [44].

As far as the anxiety experienced by patients with chronic disease, the theorystates that the underlying causes of anxiety disorders may be found in a dysfunctional cognitive bias, whereby the individual develops an over tendency to pay attention to danger, ignoring any sign of safety and/or pleasure. This fearful state of mind determines what is called 'skewed attention'. In order to modify this dysfunctional attention bringing to anxiety and stress, non-threatening gamification-supported competition is developed to train the subject away from threat/danger cues [45]. In fact, games can be used to drive positive change in health-supporting behaviors by rewarding players for accomplishing desired tasks (positive reinforcement) and by taking advantage of natural competitiveness within a friendly, mutually supportive environment. In order to avoid "nagging effect", constructive messages are provided to support individual and group motivation so as to boost user's self-esteem and reinforce positive behaviors.

The gamified approach is carried out in both passive and active ways: in the first one, by using a gamified virtual representation of their health status avatar (VHA), the patient watches (and reacts) as that persona represents their own health, enhanced also with the support of the DSS. Furthermore, the automatic measurement of biosignal data is translated into changes on the VHA. The active way represents a more direct approach of the gaming concept, i.e. this pertains to activities that the patient/user consciously comprehends to be games: challenging another user to a jogging or cycling game, a trivia competition about a particular condition, compare recent healthy lifestyles and be rewarded, etc.

Scope of the mindfulness-based gamification approach is to promote users' perception and awareness of risks while simultaneously reinforcing risk prevention user's-led behaviors through a set of easy-to learn, simple-to-use and noninvasive techniques which may promote individual's awareness, empowerment, and role modeling.

\subsection{Education}

The proposed solution educates and trains patients both to learn about their pathology and to better understand how mindfulness and the other integrated tools may help them in improving their quality of life. The concept behind our approach is that the more patients know about their condition, the more they can better manage their health, also in terms of self-management and promotion of adherence to therapeutic prescriptions and healthier behaviors (decreasing the risk behaviors and risk factors). The educational contents are shared with patients through different tools and interaction modalities (video, audio, text) in the user's mother tongue, and the educational content is tailored to the patient's background in accordance with the preliminary assessment results. Moreover, through a self-regulated learning process, patients can select the goals (challenges) they want to reach, following a gamified approach.

The patient/user can constantly access a detailed and comprehensive database/knowledge base of information about their conditions, as well as relevant updates concerning, for example, new important findings. Moreover, training and education are provided with a positive approach in accordance to the mindfulness and gamification concepts. 


\subsection{Decision Support System}

To empower patients to participate in the management of their health through applications for their disease, lifestyles and prevention monitoring, it is necessary to increase their consciousness about their health conditions and risk awareness. To this end, our solution provides users with a personal monitoring system empowered by a Decision Support System (DSS) which provides feedback about their health status, predicts health evolution and provides patients with warning alerts. The patientspecific data (e.g., biomedical, clinical, therapeutic, environmental, social data) are processed in realtime by prognostic and diagnostic algorithms based on artificial intelligence and computational models. These models and algorithms include validated medical predictive models related to the selected chronic conditions and medical guidelines (e.g., Diabetes: Archimedes [21], EAGLE [46], GDM [47] and ADA guidelines [48]; HF: Seattle HF model [20], MAGGIC [49] and European Society of Cardiology guidelines \& education). The prediction is accompanied by personalized suggestions which encourage patients to healthier behaviors and lifestyles. Moreover, the implemented DSSs generate medication reminders and warn patients on the consequences of non-adherence to the prescribed protocol.

Our solution aims to boost the use of existing validated predictive models by implementing them into a comprehensive mHealth solution supported by gamified representation and prediction of health status, and also by providing relevant recommendations based on clinical guidelines. Thus, models' outputs may become more useable and understandable to the patient thus increasing their everyday use and diffusion. The lack of large validation clinical trials and the shortage of personalized validated predictive models are problems that still need to be tackled.

\subsection{Preliminary Patient Assessment}

In order to assess in a real life context the need for a support to self-management and to lifestyle improvement, and in order to evaluate the acceptance level of our technological solution designed to relieve chronic disease burden, narrative medicine semi-structured interviews and Psycho-SocialAssessments (25 questions on physical, psychological and social well-being on a 5-item Likert scale rated on intensity/frequency of experience) were administered to a random sample of 22 patients, stratified by age and sex, attending the Chronic Care outpatient clinic at the Cardiovascular Center of Health Authority $\mathrm{n}^{\circ} 1$ Triestina (Trieste, Italy). The sample of the interviewed patients was composed by $85 \%$ males and $15 \%$ female (mean age $76 \pm 9$ ) with $8.5( \pm 3)$ years of education. $44 \%$ suffered from atrial fibrillation and $92 \%$ were in polytherapy with at least 4 medications. Furthermore, comorbidities included type II diabetes in $85 \%$ of patients, moderate to severe renal failure in $16 \%$, hypothyroidism in 20\%, and COPD (Chronic Obstructive Pulmonary Disease) in $8 \%$ of them. Patients had been previously screened and tested negative for cognitive and memory impairments. The semi-structured interviews were built on a grid which focused on the experience of illness, relationships with healthcare providers, supportive role of social network, beliefs concerning illness and adherence, self-awareness in everyday life, self-efficacy, self-report of mindful day-to-day experiences, empowerment/disempowerment, attitudes and behaviors related to adherence to therapeutic regimens and lifestyle changes, as well as technology-related attitudes and beliefs. Each narrative interview was audio-recorded and transcribed verbatim. Guidelines for grid construction in the domains of self-awareness and self-empowerment included questions from the MAAS (Mindful Attention Awareness Scale) which assesses dispositional mindfulness, (i.e., receptive awareness of and attention to what is taking place in the present) and from the PAM (Patient Activation Measure) which assesses patient knowledge, skills, and confidence for self-management [50].

The questionnaire results are summarized in $>$ Figure 2 . As it is shown, $80 \%$ of patients with a chronic cardiovascular (CV) disease have difficulties staying focused in their everyday experience; $78 \%$ reported trouble in adequately responding to shifts in the environment and $55 \%$ stated that they have trouble noticing distressing thoughts without having an emotional/physiological reaction. The majority of interviewed patients (73\%) felt disempowered as far as decision-making on health issues and treatment is concerned, and $76 \%$ of patients mistrust their self-care abilities. Interestingly, despite the advanced age of the sample, $60 \%$ of patients expressed either knowledge of, or interest in new communication technologies (e.g., mobiles, web-learning, social platforms). 
Though limited in scope, this qualitative screening sheds some light on the evolving needs for self-awareness and empowerment of patients suffering from chronic care conditions and has reinforced our approach to design the most effective solution for patients with chronic conditions.

\section{Architecture}

In order to establish an advanced system to monitor and support patients with chronic disease, the proposed solution has been designed to meet the following technical requirements, considered important aspects as stated in our introduction:

- Open architecture in order to provide a scalable system, customizable to patients affected by different chronic diseases;

- Compliance with transfer and data protocols to ensure interoperability with existing wireless devices;

- Application of semantic web approaches and standards to ensure correct sharing of information with hospital information system, and classification of patient behaviors;

- Compliance to the existing standards and regulations;

- Guarantee security and privacy protection of health and personal data.

Each module of the proposed architecture is shaped on specific patient's disease and condition states, so as to provide each user with a personalized solution. Even though the modularity can introduce limitations (e.g., increasing size and mass due to some redundancy and using standard components designed for more general applications), it allows customization, scalability and applicability to different kinds of chronicity.

Furthermore, the security, safety and interoperability of our technology are guaranteed through the compliance with existing standards. Notably, it is compliant with the ISO/IEC 27001:2005 which provide best practice recommendations on information security management, risks and controls within the context of an overall information security management system (ISMS). Moreover, data acquisition is compliant with data transfer protocols (e.g., BT4/LE, ANT+, ISO/IEEE 11073) in order to ensure integration with existing wearable devices. Patient's data are stored in accordance with privacy and safety legal requirements through the application of standard communication protocols (e.g., HL7, ICD-10 and openEHR) to ensure correct information sharing with hospital information systems and, eventually, Electronic Health Record (EHR).

In order to comply with the identified needs and requirements, the overall architecture (shown in - Figure 3) can be divided into two parts: patient side (mobile) and server side. They are both based on 3-tier architecture involving three physically separated layers: Presentation (or Client) Tier is the user interface. On the patient side, it is based on the gamification technique both for presenting and collecting data; on the server side, the view level of collected and processed data depends on the role of the logged user (i.e., healthcare professional, caregiver); Business Logic (or Application) Tier coordinates the application, processes commands, make logical decisions and evaluations, and performs calculations. It also moves and processes data between the two surrounding layers (Presentation and Data Tiers); Data (database) Tier stores and retrieves information, which is passed back to the Logic Tier for processing. Particularly, on the server side, data are permanently and safely stored, in compliance with the requirements for guaranteeing user's privacy; conversely, on the patient side, data are transitory since the database is a buffer filled with data coming from the server and the newest acquired data which are subsequently sent to the central database.

The proposed architecture consists of two parts: the former, patient side, runs on mobile device and the latter, server side, is accessible for remote monitoring.

On the patient side, a mobile application provides features and services to the patient. Through the gamification GUI module, better patient's self-care may be promoted and enhanced. Data which are automatically collected by biomedical and environmental sensors through the sensor interface, as well as self-reported data, are validated to provide real-time risk warnings/alerts whenever recorded values are out of range of safety, even when internet connection is not available. Particularly, Internet connection allows for buffered collected data to feed the DSS on the server side and to obtain remote health status monitoring and prediction of health evolution, accompanied by behavio- 
ral, lifestyle suggestions and social support provided both by caregivers and clinicians. Therapeutic reminders are also ensured in case of Internet service interruption. As sensitive data, they are being stored in the central database. However, a message reminder is locally scheduled on the Patient's device without therapy explicit references. Connection is needed to view the full text reminder with therapeutic details. A reminder engine collects all the important reminders (e.g., scheduled appointments, drugs reminders, diet, and lifestyle suggestions) and it is synchronized with the central database on the server side when Internet connection is restored. Synchronization, remote support and feedback on therapeutic compliance are only some of the features that are provided to the patient through the web services API. This API represents the interface module between the web service client interface on the patient side and the server side. The API also manages SSL encrypted traffic made up of all medical history and other sensitive data, interactive educational tools and contents, and social network interactions.

It is important to underline that no personal and/or sensitive data are stored on the patient's device. Historical data are stored on the central database. Conversely, the newly acquired data are used for urgent advices and not for consulting scope and, to this end, they are available in off-line mode until the connection is restored. Those data are temporarily stored in the buffer module after their validation. This also allows better management of a large amount of information, since data are only stored in the central database receiving and sending out necessary data on demand.

On the server side, as already briefly stated, patient's data are stored and monitored. Caregivers and healthcare professionals get patient's health information through the web application module where aggregated data and graphs, processed by the DSS module, are presented and defined in accordance with different levels of users' access. The DSS is the most important module of the business logic tier, and it is based on a relational database management system where data are stored in a disease-centric manner, as it is shown in $>$ Figure 3. The connection of this database is handled by the Data Access Layer that also grants access to its data.

The Entity-Relationship model of the database is available in the supplementary online material. The proposed database architecture allows DSS to define the proper contents to show according to the tracking progression of patient's status.

\section{Conclusions}

In conclusion, we introduced an innovative personalized technology for patients affected by chronic conditions, aimed at enhancing self-management and improving lifestyle and well-being.

The open architecture and approach offered by our solution are thought to overcome the main limitations of the present solutions available on the market, in terms of technical requirements (e.g., standards for interoperability and privacy) and humanistic and psycho-social needs (e.g., Piazza Grande). Regarding the latter, the integration of the DSS aims at improving the compliance of patients with their clinically devised pathway. With a view to strengthening the link between users and their health, the gamification approach has been introduced as an additional technique. Moreover, the enhancement of a positive psychological status, self-awareness and, consequently, quality of life is obtained through a mindfulness-based, self-empowering approach.

Particularly, the results of our preliminary assessment show the evolving needs for self-awareness and empowerment of patients suffering from chronic care conditions. The interviews' and questionnaires' results show patients' need to increase self-empowerment and self-awareness and, at the same time, their interest in new communication technologies. Nevertheless, this preliminary assessment presents several limitations (e.g. small sample, elderly patient population, high specificity and severity/complexity of disease). Even though innovative cognitive-behavioral programs may promote awareness of self and support educational and clinical interventions, careful identification of the most adequate patient population (e.g. according to age groups, complexity and/or severity of disease as well as computer literacy) is advisable so as to maximize intervention outcome. Additional research is necessary to validate this approach in a larger population and wider range of conditions. Even though technology may prove to be useful for health management, each intervention needs to be carefully built, supported and monitored by a team of well trained professionals (physicians, psychologists, nurses as well as engineers and IT specialists) to guide the integration between technol- 
ogy and the individual, so as to promote health and well-being and prevent potentially adverse events. The goal being to find the most effective and sustainable solution for patients at risk of developing, or already experiencing the burden of a chronic condition.

Once the specific needs of patients will be better identified considering also the different kinds of disease and intrinsic conditions, architecture, functionality and technical requirements will be further detailed in order to improve usefulness and usability, based on user experience.

Finally, the modularity of our solution allows for customization, scalability and applicability to different kinds of chronicity.

\section{Clinical Relevance}

Technologies for improving management of chronic conditions are growing but there are still issues to be overcome. We present the architecture of a mHealth solution that is able to meet the specific users' needs and enhance self-management skills through innovative approaches, such as mindfulness and gamification.

\section{Acknowledgments}

The work was partially supported by the Italian Ministry of Health (GR 20110235 2807).

\section{Conflict of interest}

The authors declare that they have no conflicts of interest in the research.

\section{Human Subjects Protections}

The study was performed in compliance with the World Medical Association Declaration of Helsinkion Ethical Principles for Medical Research Involving Human Subjects. 


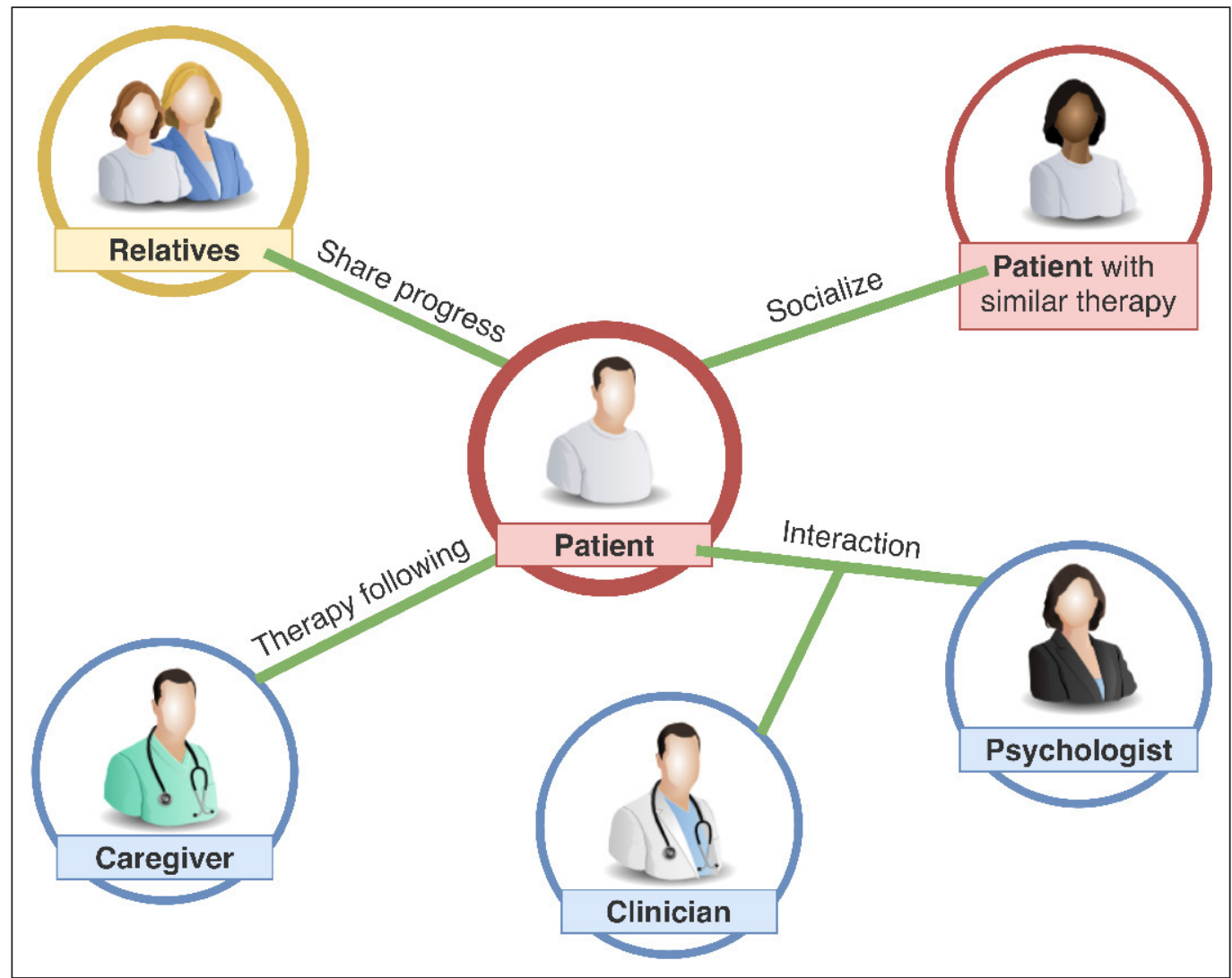

Fig. 1 Network Model of Care included in Piazza Grande

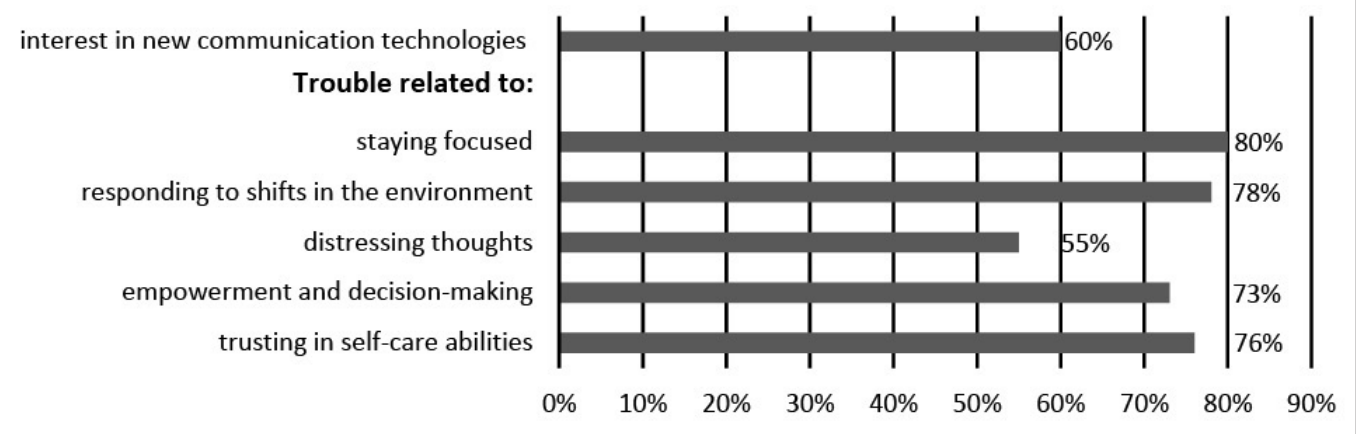

Fig. 2 Results of the preliminary assessment 


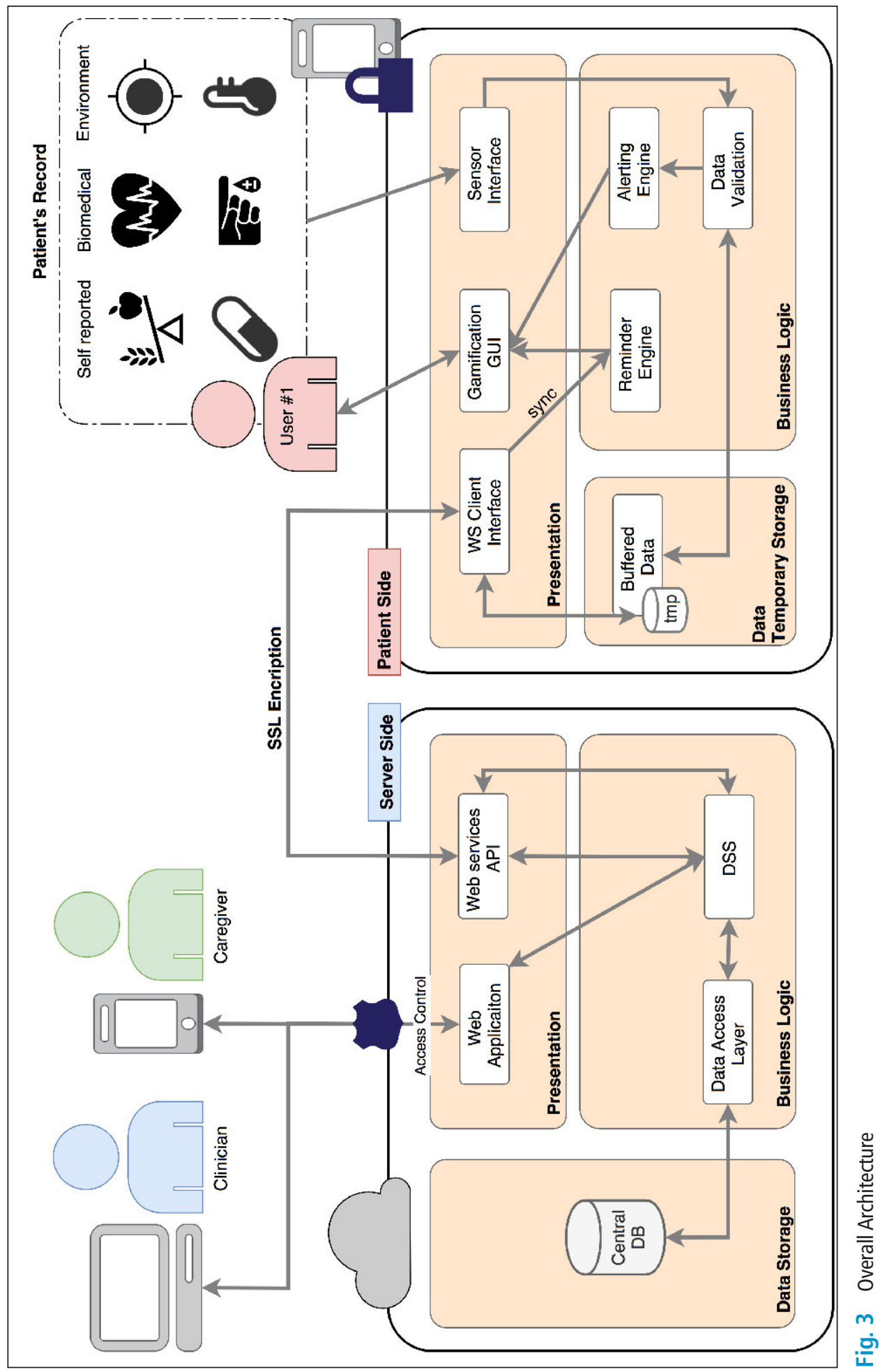

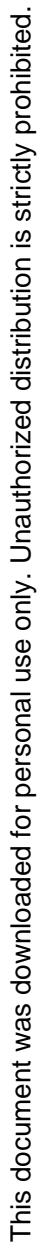




\section{References}

1. DeVol R, Bedroussian A, Charuworn A, Chatterjee A, Kim IK, Kim S, Klowden K. An unhealthy America: The economic burden of chronic disease. Santa Monica: Milken Institute; 2007.

2. Goodlin SJ, Hauptman PJ, Arnold R, Grady K, Hershberger RE, Kutner J, Masoudi F, Spertus J, Dracup K, Cleary JF, Medak R, Crispell K, Piña I, Stuart B, Whitney C, Rector T, Teno J, Renlund DG. Consensus statement: palliative and supportive care in advanced heart failure. J Card Fail 2004; 10: 200-209.

3. Chambless DL, Ollendick TH. Empirically supported psychological interventions: controversies and evidence. Annual Review of Psychology 2001; 52: 685-716.

4. Sabaté E. Adherence to long-term therapies: evidence for action. Geneva: World Health Organization; 2003.

5. Stellato K, Radini D, Pellizzari M, Pordenon M, Pletti L, Humar F, Apuzzo M, Di Lenarda A. New frontiers of people-centered integrated care for complex chronic disease. J Palliat Care Med 2015; 5(234): 2.

6. Istepanian R, Laxminarayan S, Pattichis C. M-health: emerging mobile health systems. Berlin: Springer; 2006.

7. Kay M, Santos J, Takane M. mHealth: New horizons for health through mobile technologies. Geneva: World Health Organization; 2011.

8. Luanrattana R, Win KT, Fulcher J, Iverson D. Mobile technology use in medical education. Journal of medical systems 2012; 36(1): 113-122.

9. Yang SC. Mobile applications and $4 \mathrm{G}$ wireless networks: a framework for analysis. Campus-Wide Information Systems 2012; 29(5): 344-357.

10.Marceglia S, Bonacina S, Zaccaria V, Pagliari C, Pinciroli F. How might the iPad change healthcare? Journal of the Royal Society of Medicine 2012; 105(6): 233-241.

11.Grando MA, Rozenblum R, Bates DW, editors. Information technology for patient empowerment in healthcare. Berlin: De Gruyter; 2015.

12. Sunyaev A, Dehling T, Taylor PL, Mandl KD. Availability and quality of mobile health app privacy policies. Journal of the American Medical Informatics Association 2015; 22(e1): e28-e33.

13. Chen C, Haddad D, Selsky J, Hoffman JE, Kravitz RL, Estrin DE, Sim I. Making sense of mobile health data: an open architecture to improve individual-and population-level health. Journal of medical Internet research 2012; 14(4): e112.

14.The PLOS Medicine Editors. A reality checkpoint for mobile health: three challenges to overcome. PLoS Med 2013; 10(2): e1001395.

15. Quinn P, Habbig AK, Mantovani E, De Hert P. The data protection and medical device frameworks-obstacles to the deployment of mHealth across Europe? European journal of health law 2013; 20(2): 185-204.

16. Whittaker R. Issues in mHealth: findings from key informant interviews. Journal of medical Internet research 2012; 14(5): e129.

17.Hoffman JE, Ramsey KM, Estrin D. Open architecture and standards in mobile health. In: Marsch L, Lord SE, Dallery J, editors. Behavioral Health Care and Technology. Oxford: Oxford University Press; 2015.

18. Ambroise N, Boussonnie S, Eckmann A. A smartphone application for chronic disease self-management. In: Proceedings of the 1st conference on mobile and information technologies in medicine; 2013 Nov 20; Prague, Czech Republic. 2013.

19. Kawamoto K, Houlihan CA, Balas EA, Lobach DF. Improving clinical practice using clinical decision support systems: a systematic review of trials to identify features critical to success. BMJ 2005; 330(7494): 765.

20.Levy WC, Mozaffarian D, Linker DT, Sutradhar SC, Anker SD, Cropp AB, Anand I, Maggioni A, Burton P, Sullivan MD, Pitt B, Poole-Wilson PA, Mann DL, Packer M. The seattle heart failure model: Prediction of survival in heart failure. Circulation 2006; 113(11): 1424-1433.

21.Eddy DM, Schlessinger L. Archimedes: a trial-validated model of diabetes. Diabetes Care 2003; 26(11): 3093-3101.

22. Kubitschke L, Meyer I, Müller S, Stellato K, Di Lenarda A. Digital technologies as a catalyst for change towards integrated care delivery: hype or reality?. International Journal of Reliable and Quality E-Healthcare 2016; 5(2): 31-49.

23.Navidian A, Yaghoubinia F, Ganjali A, Khoshsimaee S. The effect of self-care education on the awareness, attitude, and adherence to self-care behaviors in hospitalized patients due to heart failure with and without depression. PloS one 2015; 10(6): e0130973.

24. Finucane ML, Alhakami A, Slovic P, Johnson SM. The affect heuristic in judgments of risks and benefits. Journal of behavioral decision making 2000; 13(1): 1-17.

25. Garay-Sevilla ME, Nava LE, Malacara JM, Huerta R, de León JD, Mena A, Fajardo ME. Adherence to treatment and social support in patients with non-insulin dependent diabetes mellitus. Journal of Diabetes and its Complications 1995; 9(2): 81-86.

26. Kabat-Zinn J. Full catastrophe living: how to cope with stress, pain and illness using mindfulness meditation. New York: Dell Publishing; 1990. 
27. Kabat-Zinn J. Mindfulness based interventions in context: Past, present, and future. Clinical Psychology: Science and Practice 2003; 10(2): 144-156.

28. Baer RA, Smith GT, Allen KB. Assessment of mindfulness by self-report: the Kentucky inventory of mindfulness skills. Assessment 2004; 11(3): 191-206.

29. Teasdale JD, Segal ZV, Williams JMG, Ridgeway VA, Soulsby JM, Lau MA. Prevention of relapse/recurrence in major depression in mindfulness-based cognitive therapy. Journal of Consulting and Clinical Psychology 2000; 68: 615-623.

30.Linehan M. Cognitive-behavioral treatment for borderline personality disorder. New York: Guildford press; 1993.

31.Lau MA, Bishop SR, Segal ZV, Buis T, Anderson ND, Carlson L, Shapiro S, Carmody J, Abbey S, Devins G. The Toronto mindfulness scale: development and validation. Journal of Clinical Psychology 2006; 62(12): 1445-1467.

32. Kabat-Zinn J. Coming to our senses. New York: Hyperion; 2005.

33. Merkes M. Mindfulness-based stress reduction for people with chronic diseases. Aust J Prim Health 2000; 16(3): 200-210.

34. Clark DM. Implementing NICE guidelines for the psychological treatment of depression and anxiety disorders: The IAPT experience. International Review of Psychiatry 2011; 23(4): 318-327.

35. Williams AC, Eccleston C, Morley S. Psychological therapies for the management of chronic pain (excluding headache) in adults. Cochrane Database of Systematic Reviews 2012; 11: CD007407.

36. Hofmann SG, Sawyer AT, Witt AA, Oh D. The Effect of Mindfulness-Based Therapy on Anxiety and Depression: A Meta-Analytic Review. J Consult Clin Psychol 2010; 2: 169-183.

37. Brown KW, Ryan RM. The benefits of being present: Mindfulness and its role in psychological well-being. Journal of Personality and Social Psychology 2003; 84: 822-848.

38. Bandura A. Social learning Theory. New York: General Learning Press; 1971.

39. Di Bitonto P, Corriero N, Pesare E, Rossano V, Roselli T. Training and learning in e-Health using the gamification approach: the trainer interaction. In: Universal Access in Human-Computer Interaction. Aging and Assistive Environments. Berlin: Springer; 2014. p. 228-237.

40. Miller AS, Cafazzo JA, Seto E. A game plan: Gamification design principles in mHealth applications for chronic disease management. Health informatics journal 2014; doi: 10.1177/1460458214537511.

41. Cafazzo JA, Casselman M, Hamming N, Katzman DK, Palmert MR Design of an mHealth app for the selfmanagement of adolescent type 1 diabetes: a pilot study. Journal of medical Internet research 2012; 14(3): e70.

42. Chung BK. The power of mobile devices and patient engagement. In: Oldenburg J, editor. Engage! Transforming Healthcare Through Digital Patient Engagement. Chicago: HIMSS; 2012. p.87-98.

43. Richards D, Caldwell PH. Gamification to improve adherence to clinical treatment advice: improving adherence to clinical treatment. In: Novak D, Tulu B, Brendryen H, editors. Handbook of Research on Holistic Perspectives in Gamification for Clinical Practice. Hershey: IGI Global; 2015. p. 47-77.

44. Allam A, Kostova Z, Nakamoto K, Schulz PJ. The effect of social support features and gamification on a web-based intervention for rheumatoid arthritis patients: randomized controlled trial. Journal of Medical Internet Research 2015; 17(1): e14.

45.Dennis-TT, Egan LJ, Babkirk S, Denefrio S. For whom the bell tolls: Neurocognitive individual differences in the acute stress-reduction effects of an attention bias modification game for anxiety. Behaviour Research and Therapy 2016; 77: 105-117.

46. Mueller E, Maxion-Bergemann S, Gultyaev D, Walzer S, Freemantle N, Mathieu C, Bolinder B, Gerber R, Kvasz M, Bergemann R. Development and validation of the Economic Assessment of Glycemic Control and Long-Term Effects of diabetes (EAGLE) model. Diabetes technology \& therapeutics 2006; 8: 219-236.

47. Brown JB, Russell A, Chan W, Pedula K, Aickin M. The global diabetes model: user friendly version 3.0. Diabetes research and clinical practice. 2000; 50 (Suppl. 3): S15-S46. doi:10.1016/S0168-8227(00)00215-1.

48. American Diabetes Association. Standards of medical care in diabetes-2016 Abridged for primary care providers. Clinical Diabetes 2016; 34: 3-21.

49. Pocock SJ, Ariti CA, McMurray JJ, Maggioni A, Køber L, Squire IB, Swedberg K, Dobson J, Poppe KK, Whalley GA, Doughty RN. Predicting survival in heart failure: a risk score based on 39372 patients from 30 studies. European heart journal 2012; ehs337.

50. Hibbard JH, Stockard J, Mahoney ER, Tusler M. Development of the Patient Activation Measure (PAM): Conceptualizing and measuring activation in patients and consumers. HSR: Health Services Research 2004; 39(4): 1005-1026. 